\title{
De la psychologie et du psychologisme dans les études littéraires
}

\section{Roman Ingarden}

Traducteur : Frédéric Rogalewicz

\section{OpenEdition \\ Journals}

Édition électronique

URL : http://journals.openedition.org/alter/465

DOI : 10.4000/alter.465

ISSN : 2558-7927

Éditeur :

Association ALTER, Archives Husserl (CNRS-UMR 8547)

\section{Édition imprimée}

Date de publication : 3 décembre 2017

Pagination : 275-287

ISBN : 978-2-9550449-3-3

ISSN : $1249-8947$

\section{Référence électronique}

Roman Ingarden, «De la psychologie et du psychologisme dans les études littéraires », Alter [En ligne], 25 | 2017, mis en ligne le 01 décembre 2018, consulté le 19 avril 2019. URL : http:// journals.openedition.org/alter/465 ; DOI : 10.4000/alter.465 


\section{DE LA PSYCHOLOGIE ET DU PSYCHOLOGISME DANS LES ÉTUDES LITTÉRAIRES}

Roman Ingarden

\section{Avant-propos du traducteur}

Nous proposons ici la traduction d'un texte de Roman Ingarden rédigé en polonais sous le titre de $O$ psychologii $i$ psychologizmie w nauce o literaturze. Initialement publié en 1937 dans la revue polonaise Verticale [Pion], il fut repris dans le troisième et dernier volume des Études d'Esthétique [Studia z Estetyki] ${ }^{1}$. C'est ce texte final que nous avons traduit, sans qu'il nous ait été possible d'en vérifier la fidélité par rapport à la version initiale de 1937.

Ingarden a légèrement enrichi l'argumentaire visant le psychologisme dans la philosophie du langage, la logique et les mathématiques. Mais il a procédé à une discussion substantielle du psychologisme en esthétique et un peu en marge de ce débat, il a pu formuler des accusations contre le physicalisme. En somme, il peut être tenu pour le fondateur d'une esthétique qui s'élève contre la réduction de l'œuvre d'art à des catégories psycho-physiologiques. Dans la réflexion théorique sur la littérature en particulier, le psychologisme crée l'impression fallacieuse qu'il faut traiter l'œuvre comme un ensemble d'expériences conscientes, ce qui en élude la spécificité par rapport à toutes les autres formes de la praxis humaine. Il se fonde ainsi soit sur l'identification de l'œuvre d'art ou de l'un de ses aspects aux caractéristiques de la personnalité du créateur et de ses expériences, soit sur la réduction de l'œuvre à l'expérience per-

\footnotetext{
${ }^{1}$ Roman Ingarden, Studia $z$ estetyki, tome I [1957], Varsovie, PWN, 1966; tome II [1958],
} Varsovie, PWN, 1966 ; tome III, Varsovie, PWN, 1970. 
ceptive de son destinataire. Pour Ingarden les deux psychologismes sont également dépourvus de sens, de même qu'il serait absurde de vouloir reconstruire l'esprit d'une époque en se fondant sur l'analyse de l'œuvre d'art (autre expression du psychologisme vulgaire).

Par la critique de cette attitude courante, qui réduit à la fois l'œuvre d'art à des relations psychiques et la recherche esthétique à des études de nature psychologisante, Ingarden construit une sorte de propédeutique à la spécificité phénoménologique du mode ontologique d'existence de l'œuvre d'art et à la pluralité de ces modalités agencées en «strates » spécifiques dans le domaine de l'œuvre d'art littéraire. Tous ces thèmes sont développés dans ses deux maîtres ouvrages en la matière².

\section{DE LA PSYCHOLOGIE ET DU PSYCHOLOGISME DANS LES ÉTUDES LITTÉRAIRES}

\section{Introduction}

Il y a quelques années, je me suis déjà élevé contre le psychologisme dans l'analyse de l'œuvre littéraire. En cela, j'ai été suivi par certains savants polonais. D'autres par contre s'y sont assez clairement opposés, n'entrant pas, d'ailleurs, dans une polémique avec moi, mais avec ceux qui m'ont suivi dans cette critique. J'eus cependant l'impression que ni les uns ni les autres n'avaient tout à fait saisi les motifs réels de mon argumentation dans cette affaire.

Les partisans de l'antipsychologisme sont allés trop loin, tandis que ses adversaires contredisaient des thèses que je n'ai jamais soutenues. C'est pourquoi je désire consacrer une fois de plus mon attention à cette question.

\footnotetext{
2 Roman Ingarden, Das Literarische Kunstwerk. Eine Untersuchung aus dem Grenzgebiet der Ontologie, Logik und Literaturwissenschaft, Halle-sur-Saale, Niemeyer, 1931; L'CEuvre d'Art littéraire, trad. Philibert Secretan, avec la collaboration de N. Luchinger et B. Schwegler, Lausanne, L'Âge d'Homme, 1983, $341 \mathrm{p}$.

O poznawaniu dzieła literackiego [De la Connaissance de 1'Guvre littéraire], Lwów, Ossolineum, 1937 ; Repris dans les Studia z estetyki, tome 1 [1957], op. cit., 1966, p. 3-268, ce livre reste dans son ensemble intraduit en français à ce jour.
} 


\section{Psychologie et psychologisme}

Avant toute chose, on confond chez nous le psychologisme et la psychologie, en particulier quand il s'agit d'appliquer cette dernière à certaines questions littéraires. Par suite, on considère les adversaires $\mathrm{du}$ psychologisme comme des «ennemis » de la psychologie. Or il $s^{\prime}$ agit là de deux choses différentes. La psychologie est une science qui étudie les phénomènes et les structures mentales, elle a donc son propre champ d'étude, des méthodes heuristiques plus ou moins élaborées et des objectifs définis; elle est en cela une science non seulement entièrement légitime dans son propre champ, mais elle constitue en outre l'une des branches importantes et fondamentales de la connaissance du réel; à ce titre on ne peut ni la supprimer ni la réduire à autre chose (comme le voulaient par exemple ceux que $l^{\prime}$ on appelle les «physicalistes $»^{3}$ ). La légitimité de la psychologie ne $\mathrm{s}^{\prime}$ arrête que là où ses recherches excèdent son propre champ et se mettent à prévaloir ailleurs, par exemple dans la logique, dans la théorie de la connaissance, etc. Nous avons alors à faire face au «psychologisme » dans le sens où ce terme fut historiquement introduit par Husserl. Le " psychologisme » est un point de vue philosophique dont l'essence consiste à attribuer une caractéristique psychique à des objets non-psychiques. Psychologiste est donc celui qui considère par exemple que la Ve Symphonie de Beethoven est un ensemble d'expériences qui se sont produites dans l'âme de Beethoven au moment où celui-ci était en train de la créer. Psychologiste également celui qui affirme que le jugement dans son acceptation logique réside dans l'acte de juger ou dans le contenu de cet acte. Sur le plan de la connaissance littéraire comme partout ailleurs, le psychologisme fausse l'objet de sa recherche, notamment l'œuvre littéraire, en réduisant celle-ci à une multitude d'expériences vécues par son auteur ou son lecteur. C'est donc là une théorie toujours erronée, qui attribue à l'objet de son étude des caractéristiques qu'il ne possède pas ni ne peut posséder, et qui découle pour commencer d'une hypothèse métaphysique fausse, selon laquelle il n'existerait pas d'autre réalité

\footnotetext{
${ }^{3}$ Le terme de «physicalisme » fut créé par Rudolf Carnap en 1931 dans l'article « Die physische Sprache als Universalsprache der Wissenschaft » [ La langue de la physique comme langue universelle de la science "]. À travers lui, il s'agit de départager les sciences qui prétendent au statut de connaissance : seules celles qui se communiquent dans le langage de la physique sont des sciences authentiques. Pour Ingarden, le physicalisme considère à tort comme physiques des objets qui ne le sont pas, et qui, d'un certain point de vue, ne peuvent pas l'être. Il renvoie ainsi psychologisme et physicalisme dos à dos. [N. $d$. T.]
} 
qui ne fût ou bien psychologique ou bien physique ${ }^{4}$. Dans le domaine de la théorie littéraire, un psychologisme manifeste est du reste une théorie si clairement fausse et absurde que le fait qu'il se soit maintenu ne fût-ce qu'un instant dans la théorie philosophique générale ne se comprend à la fois que par la peur de changer sa perspective métaphysique et par les conditions particulières dans lesquelles la philosophie européenne s'est développée dans la deuxième moitié du XIXe siècle. Il y a d'ailleurs relativement peu de psychologistes qui s'assument comme tels et s'efforceraient de tirer toutes les conséquences de leur position dans des recherches spécifiques. Car les faits parlent d'eux-mêmes avec trop de force. D'ailleurs, même ces psychologistes, que ce soit dans une théorie générale ou dans leur pratique de recherche, énoncent des vérités sur telle ou telle œuvre littéraire, mais celles-ci sont en même temps inconciliables avec les principes de leurs propres positions psychologistes, lesquelles seraient trop manifestement fausses si on les rapportait à n'importe quelle expérience consciente. Or c'est justement dans cette posture masquée et inconséquente que le psychologisme est difficile à combattre et dangereux pour la recherche scientifique.

\section{L'influence du psychologisme sur la recherche}

Dans le domaine des études littéraires, le psychologisme introduit ce que j'appellerai un jargon et crée l'illusion qu'on peut traiter l'œuvre d'art en tant qu'ensemble d'expériences conscientes. Il masque ainsi la différence entre les expériences qui se rapportent à l'œuvre et l'œuvre elle-même. L'étude se déplace ainsi sur des problèmes pour le moins inessentiels, voire souvent sans aucun rapport avec l'œuvre littéraire, tandis qu'on laisse de côté les questions propres à l'étude de la littérature, ou bien qu'on les envisage sous un angle qui fausse leur sens profond. Ainsi en va-t-il, par exemple, des questions concernant la valeur artistique de l'œuvre littéraire ou de la fonction esthétique de tel ou tel facteur d'une œuvre dans sa concrétisation esthétique (dans un objet littéraire esthétique), lesquelles sont souvent soit complètement laissées dans l'ombre, soit dans le meilleur des cas déformées dans leur sens fondamental. Tout se réduit alors à la question psychologique de la qualité et de la richesse des émotions suscitées chez le lecteur sous l'influence de l'œuvre qu'il lit, ${ }^{4}$ Cette hypothèse n'est vraie que si le terme « exister » se comprend en un sens particulier et
extrêmement restreint. 
ou encore - dans un deuxième cas - au problème de la dynamique propre au vécu du récepteur de l'œuvre et du rôle joué par ses différents états dans l'expérience esthétique tout entière.

Même lorsqu'il s'agit vraiment de questions relatives au vécu du lecteur, par exemple dans l'analyse de son expérience esthétique, celles-ci sont faussées de manière significative par la conception psychologisante de l'œuvre littéraire. Si tout de même l'œuvre littéraire n'était réellement rien d'autre qu'un ensemble d'expériences vécues par l'auteur ou le lecteur, à l'évidence les vécus de connaissance de l'œuvre en général, comme ceux qui constituent sa perception esthétique spécifique, devraient alors être envisagés autrement que cela ne se passe. En premier lieu, cela reviendrait à vouloir connaître les états et les vécus psychiques d'autrui, notamment ceux de l'auteur; en second lieu, en revanche, il s'agirait d'étudier ceux des autres lecteurs ou bien les siens propres de façon réflexive lorsque le sujet connaissant est en même temps le lecteur lui-même. Ce serait évidemment absurde, mais l'évidence et le degré de cette absurdité apparaissent aussitôt que nous tentons de prendre au sérieux la compréhension psychologique de l'œuvre d'art, ne fût-ce qu'un instant, et d'en déduire toutes les conséquences.

Lequel d'entre nous serait d'accord pour dire que la connaissance d'une œuvre passe dans la lecture par des expériences réflexives et introspectives, par l'observation de nos vécus intérieurs, et non par l'étude du personnage de Zagłoba ${ }^{5}$ et de ses facéties par exemple, ou encore par l'analyse structurelle du poème Le Roi-Esprit ${ }^{6}$ ? Par ailleurs, cette inadéquation avec la manière dont nous appréhendons vraiment l'œuvre littéraire, montre à quel point la méthode psychologisante est fausse. Ne pas chercher une autre méthode nous exposerait à une énorme falsification des faits.

Mais dans la situation où le point de vue psychologisant n'est pas tout à fait conscient ou s'avance masqué, l'analyse des vécus de connaissance ou de perception esthétique de l'œuvre d'art s'expose à différentes erreurs fondamentales, notamment celle qui consiste à mêler aux vécus des aspects de l'objet perçu qui n'ont rien à voir avec eux. Les différentes valeurs esthétiques de l'objet deviennent alors

\footnotetext{
${ }^{5}$ Zagłoba est l'un des personnages principaux du roman de Sienkiewicz Par le Fer et par le Feu, rédigé dans les années 1884-1888, premier volume d'une trilogie historique extrêmement importante dans la littérature polonaise. Cf. H. Sienkiewicz, Par le Fer et par le Feu [Ogniem i mieczem], trad. L. Dyèvre et G. Gruszecka, Paris, Libretto, « Littérature étrangère », 2014. [N. $d$. T.]

${ }^{6}$ Poème historique écrit par Juliusz Słowacki dans les années 1845-1849. Cf. J. Słowacki, Le RoiEsprit [Król-Duch], trad. Charles-Edmond Chojecki [1864], s. e., disponible en ebook, traduction revue et corrigée. [N. $d$. T.]
} 
subjectives. On saisit ainsi plus ou moins bien les fonctions spécifiques de toutes les phases de l'expérience esthétique, quand on ne les omet pas, ce qui est encore pire.

Lorsqu'enfin on débarrasse l'œuvre d'art du point de vue psychologisant, mais qu'en même temps on n'en a aucun autre à mettre à la place, l'analyse de la connaissance et de la réaction psychique à l'œuvre est privée de ses jalons essentiels. On ne parvient pas à dessiner les contours des problèmes de façon suffisamment claire : on trouve dans l'expérience intérieure un flux de vécus d'une diversité et complexité importantes, dans lequel on distingue avec difficulté des différences constitutives, mais on ne connaît pas de façon positive ce à quoi ils se rapportent tous, on ne voit pas quelle fonction générale gouverne tout cet ensemble de vécus relatifs à l'œuvre littéraire ; on ne sait pas non plus quelles fonctions engage cette richesse de vécus particuliers; on ne comprend pas le lien entre eux et les phases particulières de tout le processus, de sorte que nous ne pouvons pas formuler les problèmes et orienter le cours de l'étude. On peut obtenir des résultats qui, dans ces conditions, devront non seulement se distinguer par leur remarquable contingence, mais par-delà, en ce qu'ils omettront souvent les questions justement les plus essentielles.

Je ne développerai pas ici l'influence du psychologisme sur le caractère propre des recherches épistémologiques et esthétiques dans le champ des vécus de connaissance de l'œuvre littéraire. Il faut cependant souligner que le psychologiste est enclin à considérer toutes les études épistémologiques et esthétiques, subjectivement orientées, comme des études psychologiques. De sorte que les problèmes propres à l'épistémologie, et surtout à l'épistémologie critique, n'apparaissent en général pas dans le champ de vision du chercheur, ou si cela se produit accidentellement, ils sont mal formulés. Mais développer cette question exigerait que l'on éclaircisse et détermine avec précision le propre sens de la problématique épistémologique dans son opposition aux études psychologiques, ce qui excèderait significativement le cadre de cet article?

\footnotetext{
${ }^{7}$ Je me suis efforcé de montrer quels effets produisaient les études épistémologiques sur le fondement de la psychologie dans un mémoire intitulé La Théorie psycho-physiologique de la connaissance et sa critique [Psycho-fizjologiczna teoria poznania i jej krytyka], Lwów, 1931.
} 


\section{Le lien de la psychologie avec les études littéraires}

Comme je l'ai dit, le rejet du psychologisme dans l'étude de la littérature fut mal compris autant par ceux qui, comme Manfred $\mathrm{Kridl}^{8}$ par exemple, sont d'accord pour reconnaître l'erreur de la posture psychologisante, que par ceux qui se sentent menacés dans leurs anciennes méthodes de travail par la lutte contre le psychologisme. Ces derniers défendent avec ô combien de passion parfois ces méthodes jusqu'ici dominantes et la psychologie elle-même. La « défense » de la psychologie se justifie d'autant plus que l'antipsychologisme mal compris n'affecte pas que le psychologisme: il se retourne contre la psychologie elle-même, comme on le voit chez Manfred $\mathrm{Kridl}^{9}$, qui attaque notamment la psychologie là où elle se situe dans son ordre propre, allant même jusqu'à lui refuser toute valeur scientifique. Mais même s'il est vrai que la valeur des résultats obtenus dans les recherches psychologiques n'a pas encore été définitivement démontrée par la théorie de la connaissance, même s'il est vrai que les recherches psychologiques spécifiques sont facteur de difficultés nombreuses et de possibilités d'erreurs dangereuses, même s'il est vrai, enfin, que les affirmations psychologiques que l'on retrouve dans les œuvres savantes ayant la littérature pour objet sont souvent d'une valeur très discutable et sont même parfois tout simplement fantaisistes, tout cela ne change pas le fait que la lutte contre le psychologisme n'englobe pas la lutte contre la psychologie partout où nous avons effectivement affaire à des faits et des problèmes psychologiques. Il est indiscutable que la psychologie est liée dans deux cas à l'étude de la littérature, et que dans l'un des deux elle empiète sur son champ de recherche, à savoir :

1. L'étude de la littérature est indéniablement liée à la psychologie de la création littéraire, et ainsi à la branche de la psychologie qui se consacre a) aux questions générales ayant trait aux processus mentaux de la création littéraire, b) aux recherches sur la structure psychique individuelle de l'auteur d'œuvres littéraires - ce que j'ai appelé ailleurs psychologie historique individuelle de l'écrivain. Dans un cas comme dans l'autre, nous avons affaire à des questions psychologiques qui sont tout aussi importantes et essentielles que d'autres questions relevant de psychologie générale ou même spéciale. De la même

\footnotetext{
${ }^{8}$ Manfred Kridl (1882-1957), théoricien de la littérature. [N. d. T.]

${ }^{9}$ Cf. M. Kridl, Wstęp do badań nad dziełem literackim [Introduction à l'étude de l'œuvre littéraire], Wilno, 1936. Ingarden recensera ce livre dans Pamiętnik Literacki [Le journal littéraire] en 1938. [N. d. T.]
} 
manière que l'on peut et doit faire la psychologie de l'enseignement par exemple, on peut et doit faire celle de l'écrivain ou de l'auteur. Il faut juste utiliser la psychologie à bon escient avec des méthodes de recherche idoines et un travail préparatoire et méthodologique convenable. C'est précisément à cette condition que la grande majorité de ceux qui explorent la littérature ne répondent pas lorsqu'ils mettent un point d'honneur à analyser l'expérience créatrice de l'auteur et qu'ils pensent ainsi enrichir la connaissance de son œuvre. Or il faut se rappeler qu'en conduisant de telles recherches, nous ne nous trouvons pas dans le champ d'étude de la littérature et que celles-ci conviennent mieux aux psychologues qu'aux littéraires. Ces recherches psychologiques nous font courir le danger de tomber dans les généralités et la phraséologie. Nous nous heurtons ici à une grande variété de faits difficiles à analyser, qui exigent non seulement des ressorts de subtilité et de minutie dans l'analyse, mais aussi l'invention de méthodes de recherche distinctes et adéquates.

Il y a sans doute un lien étroit entre le processus de création et l'individualité de l'auteur. Il est aussi probable que l'œuvre, si l'on peut dire, porte en elle les traces de son créateur. Aussi la connaissance du processus de création particulier et celle de la structure psychique de l'auteur peuvent-elles nous être utiles pour clarifier certains détails de l'œuvre elle-même. Réciproquement, la connaissance de certaines propriétés de cette œuvre peut nous aider à expliquer tels ou tels problèmes de psychologie de la création ou la structure mentale de l'auteur. Il faut toutefois se garder de tourner en rond en s'efforçant de connaître l'œuvre par le vécu et les propriétés psychiques de l'auteur et, inversement, l'auteur par son œuvre, laquelle serait encore inconnue dans ses caractéristiques propres. Autrement dit, les investigations psychologiques d'un côté, et les recherches littéraires de l'autre, doivent être poursuivies aussi longtemps que possible et dans une certaine mesure indépendamment les unes des autres. On ne peut utiliser les résultats obtenus par la recherche littéraire pour élucider les processus de création ou les propriétés psychiques de l'auteur qu'au moment où ceux-ci sont obtenus par l'analyse de l'œuvre seule, sans nulle référence aux résultats obtenus par ailleurs par des recherches psychologiques sur son auteur. Il en va de même lorsqu'il est question de récolter les fruits de la psychologie de l'auteur en vue d'enrichir la connaissance de la littérature. Il faut mettre en garde contre un danger fréquemment ignoré : si après un examen exhaustif de l'œuvre en tant que produit artistique, nous nous servons de cette dernière comme d'un document psychologique - et non plus alors comme d'une œuvre d'art! -, nous devons alors disposer de critères clairs et distincts par 
lesquels nous déterminerions si l'on peut considérer une propriété ou un détail de cette œuvre comme la manifestation d'une caractéristique ou d'un état psychique de l'auteur, et dans quelle mesure cela est possible. Mais peut-être n'avons-nous pas ce droit, parce qu'il ne se trouve dans l'œuvre que la réalisation d'une fonction artistique spécifique. Ces critères n'ont pas été établis jusqu'ici ${ }^{10}$ et un examen plus approfondi montrerait qu'il n'est pas facile d'en énoncer avec précision et de manière fiable. Cependant, nous passons habituellement sur ces difficultés, et c'est principalement la raison pour laquelle nous ne distinguons pas clairement l'œuvre littéraire prise en tant que produit artistique et en tant que document psychologique. Ce sont à la fois la recherche littéraire et la recherche psychologique qui ont à en pâtir, puisque cela les conduit toutes deux à des résultats faux et naïfs. Le fait même de considérer l'œuvre littéraire au sein de l'étude de la littérature comme un document psychologique constitue l'une des impostures du psychologisme et entrave grandement la recherche littéraire précise et pertinente, dont la portée se rétrécit souvent parce qu'elle n'aboutit alors qu'à des résultats qui ne tiennent aucun compte des questions spécifiquement littéraires.

Au contraire, lorsqu'il s'agit d'étudier la littérature, le fait d'utiliser des savoirs issus du champ de la psychologie de la création littéraire en général, et des informations sur l'auteur en particulier, implique le danger d'insérer dans l'œuvre littéraire différents facteurs qu'elle ne possède pas et qui n'apparaissent pas du tout non plus en elle à la libre perception de l'œuvre donnée - qui n'est alors aucunement soumise à l'influence d'informations diverses et parfois injustifiées sur l'auteur et sa vie. Introduire ces informations à l'intérieur de l'œuvre, bien qu'elles soient purement spéculatives, modifie de toute façon son contenu réel et, pire encore, non seulement cela ne l'enrichit pas et n'augmente pas sa valeur, mais cela la déforme parfois idéologiquement et esthétiquement. Par ailleurs, s'appuyer sur ce que nous savons de l'auteur et de sa vie psychique n'est utile que lorsque son ouvrage a déjà été analysé de façon exhaustive et fiable, et compris de façon synthétique. Malheureusement, trop souvent ces conditions ne sont pas remplies dans les recherches.

2. Le second groupe de questionnements psychologiques en lien avec l'étude de la littérature est constitué de problèmes ayant trait aux

\footnotetext{
10 À la suite de quoi l'intéressant livre de Stefan Baley par exemple, La personnalité créatrice du romancier Stefan Żeromski, soulève un certain nombre de doutes essentiels. [Cf. S. Baley, Osobowość twórcza Żeromskiego. Studjum z zakresu psychologji twórczości [La personnalité créatrice du romancier Stefan Żeromski. Etude de la psychologie de la création], Varsovie, Nasza Księgarnia, 1936. Ingarden recensera ce livre l'année suivante, dans Nowa Ksiażka, n 1 (1938), p. 15-16. [N. d. T.]]
} 
vécus et aux réactions des lecteurs. Un vaste champ de recherches s'ouvre dans ce domaine; au-delà de leur intérêt en tant que questionnements psychologiques, celles-ci peuvent nous être utiles pour étudier l'histoire concrète des œuvres littéraires. Elles sont susceptibles de nous fournir des matériaux pour comprendre comment se forment les courants littéraires, l'influence de la prétendue critique littéraire sur le lecteur et la façon dont il perçoit l'œuvre, etc. On a déjà engagé partiellement ce genre de tâches, notamment sous l'appellation assez malheureuse de «bibliopsychologie». On ne peut cependant pas dire que la méthodologie de ces études soit suffisamment élaborée pour le moment. L'étude plus fine de ce sujet doit être remise à plus tard. Le danger de la prise en compte de ces résultats d'études psychologiques dans le champ de la recherche littéraire n'est peut-être pas aussi grand que celle des problématiques de psychologie de la création littéraire précédemment exposées. Mais cela arrive lorsque a) l'on confond l'œuvre littéraire avec ses concrétisations et qu'en même temps b) on psychologise la concrétisation de l'œuvre littéraire en la tenant pour quelque chose qui se trouverait dans « l'âme » du lecteur ${ }^{11}$. Chacune de ces deux attitudes constitue néanmoins une manière de se fourvoyer. La concrétisation de l'œuvre littéraire est certes un objet "monosubjectif ${ }^{12}$, qui ne dépend que trop, dans ses spécificités diverses, d'un lecteur donné, de la manière particulière dont se déroule le processus de perception de l'œuvre et de la façon dont il réagit psychiquement à elle; il n'en résulte pas pour autant que la concrétisation doive être quelque chose de psychique, et en particulier une certaine composante du vécu. Si l'on étudie le vécu du lecteur lorsque celui-ci chemine avec l'œuvre, nous n'apprenons encore rien ni de cette dernière, ni de ses concrétisations particulières. Nous nous instruisons seulement sur les conditions subjectives dans lesquelles la concrétisation s'est effectuée. La compréhension de ces conditions, et simultanément la connaissance appropriée de l'œuvre littéraire, peut nous aider à tenter une interprétation hypothétique de la concrétisation ou du type de concrétisation de l'œuvre dans un temps donné et des conditions déterminées. Quand on oublie une fois encore que les recherches psychologiques dans ce domaine n'ont qu'un rôle subalterne, et qu'elles ne sauraient tenir lieu en soi d'étude ni de l'œuvre

\footnotetext{
${ }^{11}$ Plusieurs critiques de mon livre De la Connaissance de l'CEuvre littéraire, par manque de formation philosophique, ont commis l'erreur de m'imputer cette position, alors que non seulement il n'y a aucun fondement dans mon livre pour cette idée, mais, qui plus est, on y trouve des objections importantes contre ce psychologisme que j'appelle «concrétisation » de l'œuvre littéraire.

12 C'est-à-dire qu'elle n'est accessible que dans la connaissance immédiate d'un sujet singulier.
} 
littéraire ni de ses concrétisations, on va droit au psychologisme et à la confusion conceptuelle dans les études littéraires.

\section{Le problème de la psychologie dans les études littéraires}

Il y a un dernier groupe de problèmes psychologiques au sein desquels nous pénétrons dans le champ des études sur la littérature. L'œuvre littéraire n'est en elle-même rien de psychique. Cependant, dans son contenu, elle produit une strate de sujets figurés parmi lesquels apparaissent notamment des entités psycho-physiques, humaines ou animales. Bien qu'il ne s'agisse là que de personnes ou d'animaux figurés, donc d'objets d'origine intentionnelle dans leur essence ontologique, et signifiés par le texte de l'œuvre, ils ont cependant dans leur contenu une vie psychique et une spécificité individuelle qui leur sont propres, et qui doivent être analysées avec soin comme tous les autres éléments de l'œuvre. On peut donc étudier les états mentaux et la personnalité d'Ewa Pobratymska ${ }^{13}$ comme ceux de n'importe quelle personne réelle. Il faut seulement se rappeler 1) que dans ce cas le texte constitue la seule source <d'information>, 2) qu'il s'agit d'étudier un élément de l'œuvre et non pas quelque réalité indépendante, 3) enfin, qu'il s'agit d'un travail préparatoire à une connaissance approfondie de l'œuvre. Aussi nous est-il défendu, lorsque nous essayons de connaître le vécu et la spécificité individuelle des personnages figurés dans l'œuvre littéraire, de relier cette dernière à des informations que nous obtenons autrement. Ainsi, celui qui en s'appuyant sur des sources historiques obtient des informations sur le Roi Jan Kazimir, par exemple, puis analyse la Trilogie de Sienkiewicz, en particulier le personnage de Jan Kazimir, et le reconstruit non à partir du texte et de lui seul, mais à partir de ces diverses informations, fausse évidemment ce détail de l'œuvre. Et il commet une erreur encore plus grande lorsqu'il confond ces deux objets : le Roi Jan Kazimir réel et celui de la Trilogie, et qu'il rapporte à son tour au premier les résultats que l'analyse de cet élément de la Trilogie permet d'obtenir.

Naturellement, la formation psychologique du chercheur, et notamment sa connaissance des concepts fondamentaux et des affirmations de la psychologie, est nécessaire pour étudier la psychologie

${ }^{13}$ Personnage principal du roman de Stefan Żeromski Histoire du Péché [Dzieje Grzechu], paru en 1908 et adapté au cinéma par Walerian Borowczyk en 1975. Une traduction française serait bienvenue. [N. d. T.] 
des personnages représentés dans les œuvres littéraires. La psychologie générale joue ici le rôle de science auxiliaire. Mais cela ne signifie pas du tout que nous soyons autorisés, par l'analyse des vécus et spécificités psychiques individuelles des personnages, et eu égard à cet acquis psychologique jusqu'ici dominant, à placer diverses assertions parmi celles qui ne se donnent au sujet d'un personnage figuré que sur la base du texte de l'œuvre. Ce serait une attitude tout aussi illégitime que d'introduire ce type d'affirmations par l'examen psychologique d'une personne réelle. Dans le cas qui nous occupe, ce serait d'autant plus inadmissible que, par la manière dont le texte d'un ouvrage désigne une personne représentée, cette dernière peut parfaitement avoir des vécus et des propriétés psychiques qui n'arrivent pas aux personnes réelles ou qui ne sont même pas possibles en général. Cela se produit notamment partout où nous avons affaire aux déformations intentionnelles pour motifs artistiques de la vie et de la spécificité psychique individuelle des personnages - cf. certains personnages des drames de Stanisław Ignacy Witkiewicz ${ }^{14}$.

Si l'on met de côté la question de savoir si - et dans quelle mesure - les résultats d'analyses psychologiques qui portent sur des personnages figurés peuvent servir à la psychologie de vrais gens, il faut souligner deux choses :

1. Le chercheur qui limiterait l'étude du contenu d'une œuvre littéraire - ce qui arrive hélas souvent - à la seule analyse des vécus et des spécificités psychiques individuelles des personnages, et qui croirait avoir ainsi étudié la totalité de l'œuvre, déformerait cette dernière et l'appauvrirait grandement. Dans le même temps, l'étude perdrait son caractère littéraire.

2. En conséquence de quoi un tel chercheur n'aurait pas du tout accès aux problèmes de l'étude littéraire, lesquels s'ouvrent dès le moment où l'on connaît non seulement la psyché des personnages, mais également d'autres éléments de l'œuvre - donc les strates restantes, ses conséquences sur la spécificité de ses parties, etc. -, et où l'on se met à réfléchir à la fonction artistique que le personnage, et tel ou tel de ses vécus ou de ses états, remplit dans l'œuvre entière. Car toute l'analyse psychologique des personnages ne constitue en fait comme je l'ai déjà souligné - que la phase préparatoire requise pour exposer ce problème, et elle fournit la matière partielle de sa solution par l'explication de certains éléments particuliers de l'œuvre. Cette manière d'aborder la question peut aussi aider à clarifier certains problèmes littéraires, tels que définir le type d'harmonie polypho-

14 Stanisław Ignacy Witkiewicz, dit Witkacy (1885-1939). [N. d. T.] 
nique des différents éléments de l'œuvre réunis en couches, comprendre les éléments de sa composition, le type d'effets artistiques qu'elle produit - en lien avec telle configuration de la strate d'objets figurés et pas une autre -, et enfin révéler les qualités métaphysiques qui s'expriment dans l'œuvre et dans son idée.

Lorsque l'on se met à étudier les vécus psychiques des personnages figurés dans l'œuvre, non en guise de matériau pour aborder des questions littéraires, mais pour mener des études psychologiques en tant que telles, soit on se sert consciemment d'un matériau littéraire pour élucider des problèmes psychologiques - délaissant alors l'étude proprement littéraire -, soit on se leurre parce que l'on considère que ce matériau tient lieu d'étude littéraire. C'est précisément l'illusion contre laquelle il faut agir, parce que l'indulgence conduit à exclure ou du moins à déformer la problématique de l'étude littéraire, créant sur son terrain une nouvelle figure du psychologisme.

Pour finir, encore une remarque: car on peut bien étudier le monde végétal représenté dans Pan Tadeusz ${ }^{15}$, par exemple, ou dans d'autres œuvres de Mickiewicz. Est-ce que pour autant il viendrait à l'esprit de quiconque de considérer l'étude de la littérature comme une branche de la botanique ? Et est-ce que celui qui lutterait contre cette drôle d'idée serait sujet au reproche d'œuvrer contre la botanique, dont il faudrait alors prendre la «défense »? Évidemment non. Pourtant, de telles choses se produisent justement, lorsqu'au lieu des plantes nous étudions les personnages dans l'œuvre littéraire ; nous devons alors nous garder de confondre ces études avec des recherches psychologiques et, sur cette base, d'orienter les études littéraires vers la psychologie. Est-ce que cela ne rend pas suffisamment compte du niveau où se situe, chez nous, les discussions au sein de ce que l'on appelle «théorie de la littérature »?

Traduit du polonais par Frédéric Rogalewicz

\footnotetext{
${ }^{15}$ A. Mickiewicz, Pan Tadeusz, czyli Ostatni zajazd na Litwie [1834] ; Pan Tadeusz, ou la dernière expédition judiciaire en Lituanie, trad. Bernard Bourgeois, Paris, Éditions Noir sur Blanc, «Littérature étrangère », 1999. [N. $d . T$.
} 